International Mathematical Forum, 2, 2007, no. 35, 1723 - 1727

\title{
Chromatic Numbers in Some Graphs
}

\author{
Adel P. Kazemi \\ Department of Mathematics \\ University of Mazandaran \\ P. O. Box 47416-1467, Babolsar, Iran \\ a.kazemi@umz.ac.ir
}

\begin{abstract}
Let $G=(V, E)$ be a graph. A $k$-coloring of a graph $G$ is a labeling $f: V(G) \rightarrow T$, where $|T|=k$ and it is proper if the adjacent vertices have different labels. A graph is $k$-colorable if it has a proper $k$-coloring. The chromatic number $\chi(G)$ is the least $k$ such that $G$ is $k$-colorable. Here we study chromatic numbers in some kinds of Harary graphs.
\end{abstract}

\section{Mathematics Subject Classification: 05C15}

Keywords: Chromatic number; Harary's graph

\section{Introduction}

A $k$-coloring of a graph $G$ is a labeling $f: V(G) \rightarrow T$, where $|T|=k$ and it is proper if the adjacent vertices have different labels. A graph is $k$-colorable if it has a proper $k$-coloring. The chromatic number $\chi(G)$ is the least $k$ such that $G$ is $k$-colorable.[5]

One of the parameters related to chromatic number is defining number. Though we do not study it here but the interested reader can see $[1,2,3,4]$.

Given $k \leq n$, place $n$ vertices around a circle, equally spaced. If $k$ is even, form $H_{k, n}$ by making each vertex adjacent to the nearest $k / 2$ vertices in each direction around the circle. If $k$ is odd and $n$ is even, form $H_{k, n}$ by making each vertex adjacent to the nearest $(k-1) / 2$ vertices in each direction and to the diametrically opposite vertex. In each case, $H_{k, n}$ is $k$-regular. When $k$ and $n$ are both odd, index the vertices by the integers modulo $n$. Construct $H_{k, n}$ from $H_{k-1, n}$ by adding the edges $i \leftrightarrow i+(n-1) / 2$ for $0 \leq i \leq(n-1) / 2$, [1].

It is clear that $H_{2, n}=C_{n}$ and $H_{m, m+1}=K_{m+1}$. And so $\chi\left(H_{2,2 n}\right)=2$, $\chi\left(H_{2,2 n+1}\right)=3, \chi\left(H_{m, m+1}\right)=m+1$. 


\section{Main Results}

We new calculate chromatic numbers for some Harary graphs.

Lemma 1 i. Let $H=H_{2 m, n}$ or $H_{2 m+1, n}$, with $m \geq 2$. Therefore,

$$
\chi(H) \geq\left\{\begin{array}{ll}
m+2, & \text { if } m+1 \nmid n \\
m+1, & \text { if } m+1 \mid n
\end{array},\right.
$$

ii. $\chi\left(H_{3,2 n}\right) \geq \chi\left(C_{n+1}\right),(n \geq 1)$,

iii. $\chi\left(H_{3,2 n+1}\right) \geq 3$.

Proof. i. Since every $m+1$ consecutive vertices introduce a complete subgraph $K_{m+1}$, so $\chi(H) \geq \chi\left(K_{m+1}\right)=m+1$. But in this graphs the coloring function with $m+1$ colors is a congruence function to modulo $m+1$, therefore if $m+1 \nmid n$, then $\chi(H) \geq m+2$.

ii. Suppose that $V\left(H_{3,2 n}\right)=\{1,2,3, \ldots, 2 n\}$. Since the set of vertices $\{1,2, \ldots, n+$ 1 ) make a cycle with length $\mathrm{n}+1$, so $\chi\left(H_{3,2 n}\right) \geq \chi\left(C_{n+1}\right), n \geq 1$.

iii. Let $V\left(H_{3,2 n+1}\right)=\{1,2,3, \ldots, 2 n+1\}$. Since the set of vertices $\{1, n+$ $1,2 n+1\}$ makes $C_{3}$, therefore $\chi\left(H_{3,2 n+1}\right) \geq 3$.

Theorem 2 For every $n=(m+1) k+r$, where $k \geq r$, we have

$$
\chi\left(H_{2 m, n}\right)=\left\{\begin{array}{l}
m+1, \quad \text { if } r=0 \\
m+2, \quad \text { otherwise }
\end{array} .\right.
$$

Proof. We have $n=(m+1)(k-r)+r(m+2)$, when $k \geq r$. If $m+1 \mid n$, then the coloring function $f$ to modulo $m+1$, i.e.,

$$
f(i)=j, \quad i \equiv j(\bmod m+1),(1 \leq i \leq n),
$$

is a proper coloring. So $\chi=m+1$, by Lemma 1. Now, we suppose that $m+1 \nmid n$. The coloring function $f$ with criterion

$$
\begin{aligned}
& f(i)=j, \quad i \equiv j(\bmod m+1), \quad 1 \leq i \leq(k-1)(m+1), \\
& f(i)=j, \quad i \equiv j \quad(\bmod m+2), \quad(k-1)(m+1)+1 \leq i \leq n,
\end{aligned}
$$

and Lemma 1 imply $\chi=m+2$.

We know that if $n \geq m(m+1)$, then

$$
\chi\left(H_{2 m, n}\right)=\left\{\begin{array}{lc}
m+1, & \text { if } m+1 \mid n \\
m+2, & \text { otherwise }
\end{array},\right.
$$

by [5]. Now, we determine $\chi\left(H_{2 m, n}\right)$ for some $n<m(m+1)$. 
Theorem 3 If $n=m(m+1)-i$ for $2 \leq i \leq m$ and $m \geq\lceil(1+\sqrt{4 i+5}) / 2\rceil$, then $\chi\left(H_{2 m, n}\right)=m+2$.

Proof. The assumptions $n=(i-2)(m+1)+(m-i+1)(m+2)$ and $n \geq 2 m+1$ imply $m^{2}-m-i-1 \geq 0$. Since $m+1 \nmid n$ therefore $\chi \geq m+2$. Now, the proper coloring function $f$ with criterion

$$
\begin{aligned}
& f(i)=j, \quad i \equiv j(\bmod m+1), \quad 1 \leq i \leq(i-2)(m+1), \\
& f(i)=j, \quad i \equiv j(\bmod m+2), \quad(i-2)(m+1)+1 \leq i \leq n,
\end{aligned}
$$

implies $\chi\left(H_{2 m, n}\right)=m+2$.

Theorem 4 For every $n \geq m+1$, we have

$$
\chi\left(H_{2 m+1,2 n}\right)=\left\{\begin{array}{l}
m+1, \quad \text { if } 2 n=(m+1) t \text { and } t \text { is odd } \\
m+2, \quad \text { if } 2 n=(m+2) t, t \text { is odd and } m+1 \nmid t
\end{array} .\right.
$$

Proof. Suppose that $2 n=(m+1) t$ and $t$ is odd. The coloring function $f$ to modulo $m+1$ is a proper coloring. Because $n \not \equiv 0(\bmod m+1)$ concludes $f(i+n) \neq f(i)$ for $1 \leq i \leq n$. Therefore $\chi\left(H_{2 m+1,2 n}\right)=m+1$.

Now, we suppose that $2 n=(m+2) t, t$ is odd and $m+1 \nmid t$. The coloring function $f$ to modulo $m+2$ is a proper coloring. Because $n \not \equiv 0(\bmod m+2)$ concludes $f(i+n) \neq f(i)$ for $1 \leq i \leq n$. And since $m+1 \nmid 2 n$ so $\chi=m+2$ by Lemma 1 .

As an immediately result, we have.

Corollary 5 If $2 n=(m+1)(m+2) t$ such that both of which $t$ and $m$ are odd, then $\chi\left(H_{2 m+1,2 n}\right)=m+1$.

$$
\begin{aligned}
& \text { If } n=(m+1) k+r \text { where } 1 \leq r \leq m \text {, then } \\
& \qquad \chi\left(H_{2 m+1,2 n}\right)=m+1 \Leftrightarrow m \text { is odd and } r=(m+1) / 2 .
\end{aligned}
$$

Proof. We know $\chi \geq m+1$. But $\chi=m+1$ if and only if $n \not \equiv 0(\bmod$ $m+1)$ and $f(2 n)=m+1$. Since $n \not \equiv 0(\bmod m+1)$ and $f(2 n)=f(2 r)$, so $\chi=m+1$ if and only if $2 r=m+1$.

Obviously, $\chi\left(H_{2 m+1,2 n}\right) \geq m+2$ if $m$ is even or if $m$ is odd and $r \neq(m+1) / 2$, by the Proposition 7.

Theorem 6 For every $n \geq 2, \chi\left(H_{3,2 n+1}\right)=3$. 
Proof. Let $V=\{1,2,3, \ldots, 2 n+1\}$, we know $\chi \geq 3$, by Lemma 1 . We, now, consider three cases as follows.

Case 1. $2 n+1=4 k+5, k \geq 0,3 \mid n$.

The coloring function $f$ with criterion

$$
\begin{gathered}
f(i)=\delta_{i}, \text { where } \delta_{i} \equiv\left\{\begin{array}{c}
i(\bmod 3), \quad 1 \leq i \leq n \\
i+1(\bmod 3), \quad n+1 \leq i \leq 2 n
\end{array} \quad \text { and } 1 \leq \delta_{i} \leq 3,\right. \\
f(2 n+1)=3,
\end{gathered}
$$

is a proper coloring with three colors. Because $f(n+1)=2 \neq f(2 n+1)$ and since $3 \mid n$ therefore $f(i+n)=f(i+1) \neq f(i), 1 \leq i \leq n$.

Case 2. $2 n+1=4 k+5, k \geq 0,3 \nmid n=2 k+2$.

Let $n=3 t+r, 1 \leq r \leq 2$. The coloring function $f$ to modulo 3 is a proper coloring. Because $f(i+n)=f(i+r) \neq f(i)$ for $1 \leq i \leq n$. Also, $f(n+1)=r+1 \neq f(2 r+1)=f(2 n+1)$.

Case 3. $2 n+1=4 k+7, k \geq 0$.

The coloring function $f$ with criterion

$$
f(i)=j, \quad i \equiv j(\bmod 2), \quad 1 \leq i \leq 2 n, 1 \leq j \leq 2, \quad f(2 n+1)=3
$$

is a proper coloring with three colors. Because $f(i+n)=f(i+1) \neq f(i)$ for $1 \leq i \leq n$ and $f(n+1)=2 \neq f(2 n+1)$. So, we conclude $\chi\left(H_{3,2 n+1}\right)=3$, for $n \geq 2$.

Theorem 7 If $m+1 \nmid n$. Then

$\chi\left(H_{2 m+1,2 n+1}\right)=\left\{\begin{array}{cc}m+2, & m \text { is even }, n \equiv \frac{m}{2}(\bmod m+2), n \neq \frac{m}{2}(\bmod m+1) \\ m+2, & m \text { is odd } n \equiv \frac{m+1}{2}(\bmod m+2) \\ m+1, & m \text { is even } n \equiv \frac{m}{2}(\bmod m+1)\end{array}\right.$.

Proof. In the two first cases, $m+1 \nmid 2 n+1$ so $\chi\left(H_{2 m+1,2 n+1}\right) \geq m+2$. Now, it is clear that the coloring function to modulo $m+2$ is proper. So, $\chi=m+2$. But in the last case, the coloring function to modulo $m+1$ is proper and so $\chi=m+1$.

We note that if $m \geq 3$, then $3 m+2<m(m+1)$ and since $m+1 \nmid n=3 m+2$ so $\chi \geq m+2$. But if $f$ is a coloring function with $m+2$ colors, then $f(i)=i$, $1 \leq i \leq m+1$. For the remained vertices the coloring classes are

$$
\begin{gathered}
c l(m+i)=\{m+2\} \cup\{j \mid 1 \leq j \leq i-1\}, \text { for } i=2,3, \ldots, m+2, \\
c l(2 m+i)=\{j \mid i-1 \leq j \leq m+2\}, \text { for } i=3,4, \ldots, m+2 .
\end{gathered}
$$

We end this note with th following question.

Question. If $m \geq 3$, then one can say $\chi\left(H_{2 m, 3 m+2}\right) \neq m+2$ ? 


\section{References}

[1] D. Donovan, E. S. Mahmoodian, R. Colin and P. Street, Defining sets in combinatorices: a survay, London Mathematical Society Lecture Note Series 307 (2003).

[2] E. S. Mahmoodian, R. Naserasr, M. Zaker, Defining sets in vertex colorings of graphs and Latin rectangles. 15th British Combinatorial Conference (Stiriling, 1995). Discrete Math. 167/168 (1997), 451-460.

[3] D. Mojdeh, On the defining spectrum of $k$-regular graphs with $k-1$ colors. The Journal of Prime Research in Mathematics, Vol. 1, No. 1 (2005), 118135 .

[4] A. P. Street, Defining sets for block designs; an update, in: C. J. Colbourn, E. S. Mahmoodian (Eds), Combinatorics advances, Mathematics and its applications Kluwer academic Publishers, Dordrecht, (1995) 307-320.

[5] D. B. West, Introduction to gragh theory, (2nd edition), Prentice Hall USA (2001).

Received: November 30, 2006 